\title{
Locus Chemotherapy in Cancer Angiogenesis
}

\section{Ziv Radisavljevic*}

Department of Surgery, Brigham and Women's Hospital, Harvard Medical School, Boston, Massachusetts, USA

\begin{abstract}
A cancer angiogenesias is a complex system. Its robustness is supported by the signaling elements from cancer hypoxic microenvironment. The signaling angiogenic networks have nodal organization of its elements. The AKT is crucial macromolecule in the nodal angiogenic system of solid cancers, leukemia, lymphoma, plasmocytoma and myeloma. Targeting AKT locus by the locus chemotherapy, angiogenesis and all cancer system will fail. The AKT dephosphorylation phenomenon is induced by the redox balance change, causing conversion of the positive loops into normal feedback loops and leading to abolishment of cancer angiogenesis and cancer progression.
\end{abstract}

\section{Keywords: Locus chemotherapy; Angiogenesis; Cancer}

A cancer angiogenic signaling networks are crucial process for malignant progression in solid cancers and hematological malignancies such as leukemia, lymphoma, plasmocytoma and myeloma [1]. The signaling interactome governing that process is very complex in nodal organization of the signaling genes [2]. Understanding that process at genomic and molecular level will solve many dilemmas for cancer progression, therapies, multidrug resistance [3], and led to the development of the successful locus chemotherapy. The terminology of locus chemotherapy was recently introduced in complex and robust cancer system and in cancer angiogenesis [1-4].

An angiogenic nodal organization of the signaling genes in cancer interactome is unique with specific pattern, where AKT gene represents main locus, governing connected genes in signaling interactome. The AKT gene, as an angiogenic locus is downstream of vascular endothelial growth factor (VEGF), hypoxia inducible factor 1 alpha (HIF-1 $\alpha$ ), $\mathrm{H}_{2} \mathrm{O}_{2}$, and hepatocyte growth factor (HGF) and upstream of mTOR and NOS genes [1]. The AKT is in the center of the upstream activating signals and downstream rebound activating signals of the cancer positive feedback signaling loops [2]. If that locus is targeted by oxidant/antioxidant balance change [4], angiogenic robustness and cancer progression will irreversibly fail $[1,2]$. However, if the VEGF is targeted by the bevacizumab, a monoclonal antibody against circulating vascular endothelial growth factor A (VEGF-A) in breast cancer [5], only that signaling macromolecule will be blocked, but rest of the activating signals from the cancer hypoxic microenvironment will freely hyper stimulate the AKT and cancer angiogenesis will progress further [1], causing the absence of a benefit for overall survival [5]. Progression of disease is observed in colorectal cancer after use of the Bevacizumab plus fluoropyrimidine-based chemotherapy, a standard first-line treatment, and bevacizumab-naive second-line in metastatic colorectal cancer in KRAS wild-type and mutant KRAS tumors [6] as well as in metastatic breast cancer and non-small cell lung cancers [7]. Maintenance of VEGF inhibition with bevacizumab as a first line therapy leads to partial response because of the partial inhibition of the signaling elements in the signaling interactome. This is a phenomenon of the peripheral blocking of one signaling molecule such as VEGF instead of targeting signaling AKT locus as a key element in controlling angiogenic signaling networks.

A cancer angiogenic signaling interactome is controlled by the AKT locus. The locus got the power by amplified stimuli from the cancer hypoxic microenvironment and from feedback rebound signals [2]. The angiogenic robustness can be regulated by the redox balance in the cancer cell $[1,4]$, causing conversion of the positive signaling loops into negative feedback loops [2], halting cancer angiogenesis [1] and cancer progression [2,4]. Targeting AKT locus with locus chemotherapy represents successful novel approach to suppress tumor angiogenesis, cancer progression and cancer multidrug resistance at the same time [1-4].

\section{References}

1. Radisavljevic $Z$ (2013) AKT as locus of cancer angiogenic robustness and fragility. J Cell Physiol 228: 21-24.

2. Radisavljevic $Z$ (2013) AKT as locus of cancer positive feedback loops and extreme robustness. J Cell Physiol 228: 522-524.

3. Radisavljevic Z (2013c) AKT as locus of cancer multidrug resistance and fragility. J Cell Physiol 228: 671-674.

4. Radisavljevic $Z$ (2008) AKT as locus of fragility in robust cancer system. J Cell Biochem 104: 2071-2077.

5. Montero AJ, Vogel C (2012) Fighting fire with fire: rekindling the bevacizumab debate. N Engl J Med 366: 374-375.

6. Kubicka S, Greil R, Andre T, Bennouna J, Sastre J, et al. (2013) Bevacizumab plus chemotherapy continued beyond first progression in patients with metastatic colorectal cancer previously treated with bevacizumab plus chemotherapy: ML18147 study KRAS subgroup findings. Ann Oncol 24: 23422349.

7. Bennouna J, Sastre J, Arnold D, Österlund P, Greil R, et al. (2013) Continuation of bevacizumab after first progression in metastatic colorectal cancer (ML18147): a randomised phase 3 trial. Lancet Oncol 14: 29-37.

*Corresponding author: Ziv Radisavljevic, Department of Surgery, Brigham and Women's Hospital, Harvard Medical School, Boston, Massachusetts, USA, E-mail: zradisavljevic@rics.bwh.harvard.edu

Received November 18, 2013; Accepted November 19, 2013; Published November 21, 2013

Citation: Radisavljevic Z (2013) Locus Chemotherapy in Cancer Angiogenesis. Biol Syst Open Access 2: e106. doi:10.4172/2329-6577.1000e106

Copyright: (c) 2013 Radisavljevic Z. This is an open-access article distributed under the terms of the Creative Commons Attribution License, which permits unrestricted use, distribution, and reproduction in any medium, provided the original author and source are credited. 